\title{
Molecular Imaging Shared Resource
}

National Cancer Institute

\section{Source}

National Cancer Institute. Molecular Imaging Shared Resource. NCI Thesaurus. Code C39461.

The Molecular Imaging Shared Resource develops imaging reagents and methods that can be widely applied, develops imaging instruments, translates imaging methods to human studies, identifies biological pathways and organs for disease-specific change targeting. 